\title{
Parametric Characterization of Truncated Circular Flattened Gaussian Beams
}

\author{
Redouane Lamsoudi, Mohammed Ibn Chaikh \\ Laboratory of Physics of Condensed Matter, Physics Department, Faculty of Science, Chouaïb Doukkali University, El Jadida, Morocco
}

Email address:

lamsoudi@gmail.com (R. Lamsoudi)

To cite this article:

Redouane Lamsoudi, Mohammed Ibn Chaikh. Parametric Characterization of Truncated Circular Flattened Gaussian Beams. American Journal of Optics and Photonic. Vol. 3, No. 1, 2015, pp. 1-4. doi: 10.11648/j.ajop.20150301.11

\begin{abstract}
Based on the truncated second-order moments definition, the generalized factor of the circular flattened Gaussian beams (CFGB) in the cylindrical coordinate system through a hard-edged circular aperture is derived. Three special cases have been obtained from the closed-form expression for the generalized factor of the truncated CFGB, the non-truncated CFGB, the truncated and non-truncated Gaussian beams. The power fraction of the CFGB is calculated analytically and illustrated numerically.
\end{abstract}

Keywords: Generalized Factor, Power Fraction, Circular Flattened Gaussian Beams

\section{Introduction}

In many applications, such as material thermal processing, inertial confinement fusion, second-harmonic generation, electron acceleration, and optical communication, a laser beam with a flat-topped spatial profile is required $[1,2]$. This allows one to make better use of all the energy that is present in the laser beam or to make use of the entire volume. Several theoretical models have been proposed to describe a coherent flat-topped beam of circular symmetry [3, 4]. The desired uniform intensity distribution leads one to the study of Flattened Gaussian Beams (FGBs) or Super Gaussian Beams (SGBs). Flat-top laser beams can be realized by converting a Gaussian beam from a single transverse mode laser using an optical beam shaper [5]. Several approaches have been reported for the design of flat-top beam shaping devices. The most straightforward method is to truncate or attenuate the input Gaussian beam using a neutral density filter with a proper transversal transmittance profile [5]. The drawback of this approach is its poor energy efficiency. To improve the beam shaping efficiency, both reflective and refractive optical systems have been considered, resulting in the requirement of sophisticated optical surfaces that are difficult to fabricate and high beam shaper fabrication costs [6].

Recently, the characterization of laser beams has been the subject of many research works [7, 8]. In the last decade, many parameters and factors have been introduced in literature to describe different laser beams in practice. Among those parameters, we found the power in bucket (PIB), the $\mathrm{M}_{\mathrm{G}}^{2}$ factor which is used to predict the laser beam quality [9, 10]

The analytical calculation of the power entering through an aperture (the power fraction) and the generalized beam propagation factor for different laser beams rectangular symmetry has been based on the definition of the moments of intensity of order 2. However, we must appeal to the definition of moment of intensity truncated [11] when the $\mathrm{ABCD}$ optical system has an opening. In the literature, the laser beams truncated Gauss beams, cosh-Gauss beams and Hermite-cosh-Gaussian beams admit analytical solutions [12, 13].

In this step of work, we determine the closed-form expression for the power fraction (PIB) and the generalized beam propagation factor for truncated circular flattened Gaussian beams. These two last parameters are already determined in an approximate tone [14]. The truncated and no truncated Gaussian beams can be deduced as a particular case of the considered beams when $\mathrm{N}=1$.

\section{Power Fraction of Circular Flattened Gaussian Beams}

The electric field of circular flattened Gaussian beams at $\mathrm{z}=0$ can be expressed as the following finite sum of Gaussian modes 


$$
E(r, 0)=\sum_{n=1}^{N} b_{n} \exp \left(-\frac{n r^{2}}{w^{2}}\right)
$$

Here $w$ is the waist width of the circular flattened Gaussian beams, the amplitude parameter

$$
b_{n}=\frac{(-1)^{n-1}}{N}\left(\begin{array}{l}
N \\
n
\end{array}\right) \text {. }
$$

Within Eq.(2), $\left(\begin{array}{l}N \\ n\end{array}\right)$ denotes a binomial coefficient and $N$ $(N=1,2,3, \ldots)$ is the order of the flattened Gaussian beams. When $N=1$, Eq.(1) reduces to a Gaussian beam.

The power fraction $P$ is given as:

$$
P=\frac{I_{0 N}^{a}}{I_{0 N}^{\infty}}
$$

Where, the total power entering through the aperture of $a$ radius is defined by

$$
I_{0}^{a}=\int_{0}^{a} \int_{0}^{2 \pi}\left|E_{n}(r, \theta, 0)\right|^{2} r d r d \theta
$$

And, the total power of the beam through out the space.

$$
I_{0}^{\infty}=\int_{0}^{+\infty} \int_{0}^{2 \pi}\left|E_{n}(r, \theta, 0)\right|^{2} r d r d \theta
$$

The substitution from Eq. (1) into Eq. (3b) and after some algebraic manipulation, we obtain the expression of the total power

$$
I_{0}^{a}=\pi \omega^{2} \sum_{n=1}^{N} \sum_{m=1}^{N} b_{n} b_{m}\left(\frac{\mathcal{P}\left(2 \mathrm{t}_{1}^{2} / 2\right)}{n+m}\right)
$$

Where, the probability integral of the $\chi^{2}$-distribution defined by $[15,16]$

$$
\mathcal{P}\left(\chi^{2} / v\right)=\frac{1}{2^{v / 2} \Gamma\left(\frac{v}{2}\right)} \int_{0}^{\chi^{2}} \mathrm{t}^{\frac{v}{2}-1} \mathrm{e}^{-\frac{\mathrm{t}}{2}} \mathrm{dt}
$$

And

$$
t_{1}=\sqrt{n+m} \alpha
$$

The truncation parameter

$$
\alpha=\frac{a}{w}
$$

By letting $a \rightarrow \infty$ and, use of the property of the probability function

$$
\lim _{\chi \rightarrow \infty} \mathcal{P}\left(\chi^{2} / \xi\right)=1
$$

One obtains

$$
I_{0}^{\infty}=\pi \omega^{2} \sum_{n=1}^{N} \sum_{m=1}^{N} b_{n} b_{m}\left(\frac{1}{n+m}\right)
$$

Finally, Eq. (3a) can be written as

$$
P=\frac{\sum_{n=1}^{N} \sum_{m=1}^{N} b_{n} b_{m}\left(\frac{\mathcal{P}\left(2 \mathrm{t}_{1}^{2} / 2\right)}{n+m}\right)}{\sum_{n=1}^{N} \sum_{m=1}^{N} b_{n} b_{m}\left(\frac{1}{n+m}\right)}
$$

This equation is a closed-form of the power fraction of circular flattened Gaussian beams. To illustrate this result.Fig. 1 describes the variation of $P$ versus truncation parameter $\alpha$ for some values of $N$.

From this formula, we can deduce two limiting cases:

- for an infinitely large aperture. in this case the Eq.(8) is reduced to

$$
P=1
$$

- When $\mathrm{N}=1$ the expression of $P$ reduced to the $P_{\text {Gaussian }}$.

$$
P_{\text {Gaussian }}=\mathcal{P}\left(4 \alpha^{2} / 2\right)
$$

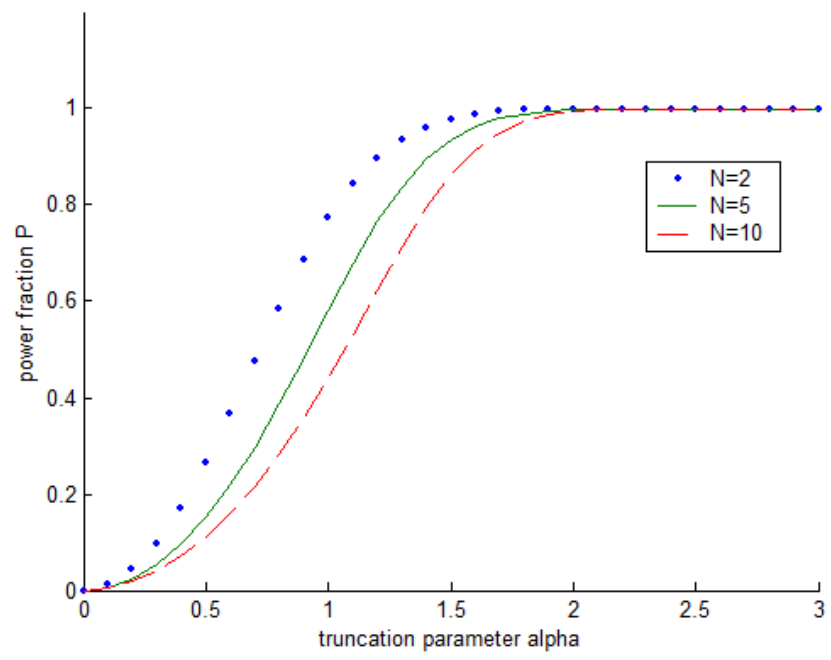

Fig. 1. The power fraction $P$ as a function of truncation parameter $\alpha$ for various order $N$.

\section{3. $M_{G}^{2}$-Factor of Truncated Circular Flattened Gaussian Beams}

Following the Refs [7], we define the second-order irradiance $\left\langle r^{2}\right\rangle$ in spatial domain, $\left\langle p^{2}\right\rangle$ in frequency domain and the cross second moment $\langle\mathrm{r} p\rangle$ of a hard-edged diffracted read

$$
\left\langle r^{2}\right\rangle=\frac{1}{I_{0}^{a}} \int_{0}^{a} \int_{0}^{2 \pi} r^{2}|E(r, \theta, 0)|^{2} r d r d \theta
$$

The prime indicate derivation with respect to $r, K$ is the wave number, and ${ }^{*}$ is the complex conjugate.
The generalized beam propagation factor $M_{G}^{2}$-factor reads in the cylindrical coordinate system 


$$
M_{G}^{2}=K \sqrt{\left\langle r^{2}\right\rangle\left\langle\mathrm{p}^{2}\right\rangle-\langle r \mathrm{p}\rangle^{2}} .
$$

Substituting from Eq. (1) into Eq. (11) can be written as

$$
\left\langle\mathrm{p}^{2}\right\rangle=\frac{\pi}{K^{2} I_{0}^{a}} \sum_{n=1}^{N} \sum_{m=1}^{N} 4 n m b_{n} b_{m}\left(\frac{\mathcal{P}\left(2 \mathrm{t}_{1}^{2} / 4\right)}{(n+m)^{2}}\right)+\frac{32 \pi}{3 K^{2} I_{0}^{a}}|\mathrm{E}(a, 0)|^{2}
$$

Where

$$
|E(a, 0)|^{2}=\sum_{n=1}^{N} \sum_{m=1}^{N} b_{n} b_{m} \exp \left(-(n+m) \alpha^{2}\right)
$$

Gaussian field distribution is real valued in the waist plane.

Finally, the analytical expression of $M_{G}^{2}$-factor can be written as

Note that $\langle\mathrm{r} p\rangle$ vanishes since the Circular Flattened

$$
M_{G}^{2}=\frac{\sqrt{\sum_{\mathrm{n}=1}^{\mathrm{N}} \sum_{\mathrm{m}=1}^{\mathrm{N}} \mathrm{b}_{\mathrm{n}} \mathrm{b}_{\mathrm{m}}\left(\frac{\mathcal{P}\left(2 \mathrm{t}_{1}^{2} / 4\right)}{(\mathrm{n}+\mathrm{m})^{2}}\right)\left(\sum_{\mathrm{n}=1}^{\mathrm{N}} \sum_{\mathrm{m}=1}^{\mathrm{N}} 4 \mathrm{nmb}_{\mathrm{n}} \mathrm{b}_{\mathrm{m}}\left(\frac{\mathcal{P}\left(2 \mathrm{t}_{1}^{2} / 4\right)}{(\mathrm{n}+\mathrm{m})^{2}}\right)+\frac{32}{3} \sum_{\mathrm{n}=1}^{\mathrm{N}} \sum_{\mathrm{n}=1}^{\mathrm{N}} \mathrm{b}_{\mathrm{n}} \mathrm{b}_{\mathrm{m}} \exp -\mathrm{t}_{1}^{2}\right)}}{\sum_{\mathrm{n}=1}^{\mathrm{N}} \sum_{\mathrm{m}=1}^{\mathrm{N}} \mathrm{b}_{\mathrm{n}} \mathrm{b}_{\mathrm{m}}\left(\frac{\mathcal{P (}\left(2 \mathrm{t}_{1}^{2} / 2\right)}{\mathrm{n}+\mathrm{m}}\right)}
$$

From this formula, we can deduce two limiting cases:

- for an infinitely large aperture, in this case the Eq.(18) is reduced to

$$
M^{2}=\lim _{\alpha \rightarrow \infty} M_{G}^{2}=\frac{\sqrt{\sum_{\mathrm{n}=1}^{\mathrm{N}} \sum_{\mathrm{m}=1}^{\mathrm{N}} \mathrm{b}_{\mathrm{n}} \mathrm{b}_{\mathrm{m}}\left(\frac{1}{(\mathrm{n}+\mathrm{m})^{2}}\right)\left(\sum_{\mathrm{n}=1}^{\mathrm{N}} \sum_{\mathrm{m}=1}^{\mathrm{N}} 4 \mathrm{nmb}_{\mathrm{n}} \mathrm{b}_{\mathrm{m}}\left(\frac{1}{(\mathrm{n}+\mathrm{m})^{2}}\right)\right)}}{\sum_{\mathrm{n}=1}^{\mathrm{N}} \sum_{\mathrm{m}=1}^{\mathrm{N}} \mathrm{b}_{\mathrm{n}} \mathrm{b}_{\mathrm{m}}\left(\frac{1}{\mathrm{n}+\mathrm{m}}\right)}
$$

- If $N=1$ corresponding to Gaussian beams. Using the relation between the probability integral of the $\chi^{2}$ distribution and the incomplete gamma function, and the recurrence relation for the incomplete gamma function Eq.(18) simplifies to

$$
M_{G}^{2}=\frac{\sqrt{\left(1-\mathrm{e}^{-2 \alpha^{2}}-2 \alpha^{2} \mathrm{e}^{-2 \alpha^{2}}\right)\left(1+\frac{29}{3} \mathrm{e}^{-2 \alpha^{2}}-2 \alpha^{2} \mathrm{e}^{-2 \alpha^{2}}\right)}}{1-\mathrm{e}^{-2 \alpha^{2}}}
$$
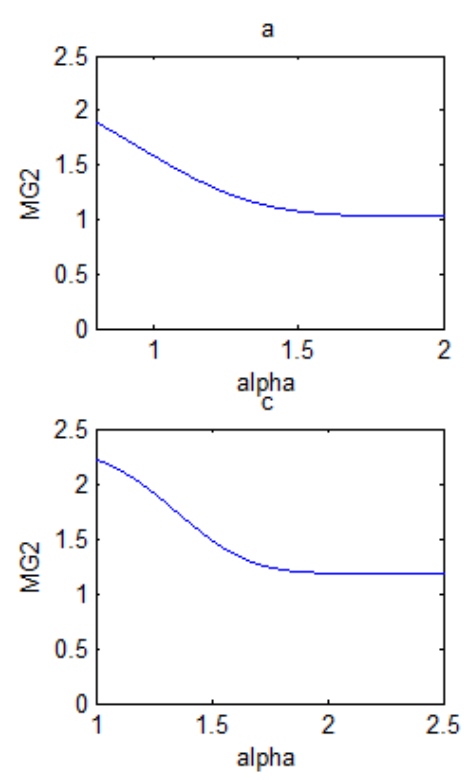

Fig. 2. $M_{G}^{2}$ - factor of a circular flattened Gaussian beams versus $\alpha$ with (a) $N=2$, (b) $N=3$, (c) $N=4,(d) N=5$.

These figures show that for all values of beam order, the $M_{G}^{2}$ - factor decreases with $\alpha$ and tends to the no truncated $M^{2}$-factor. So, for $N=2$, the value of $M^{2}$ is 1.0307 . From these figures, we can determine the values of $M^{2}$ which are respectively: $1.1353,1.1833,1.2370$ for $N=5,7$, and 10 . Also, we can determine from our analytical expression of the
The expression (18) is a closed-form of $M_{G}^{2}$ - factor of the circular flattened Gaussian beam passing through a hardedged aperture. To illustrate Eq.(18), we present in Figs. 2 , the $M_{G}^{2}$ - factor versus the truncation parameter $\alpha$ for which the power fraction, for various values of $N$.
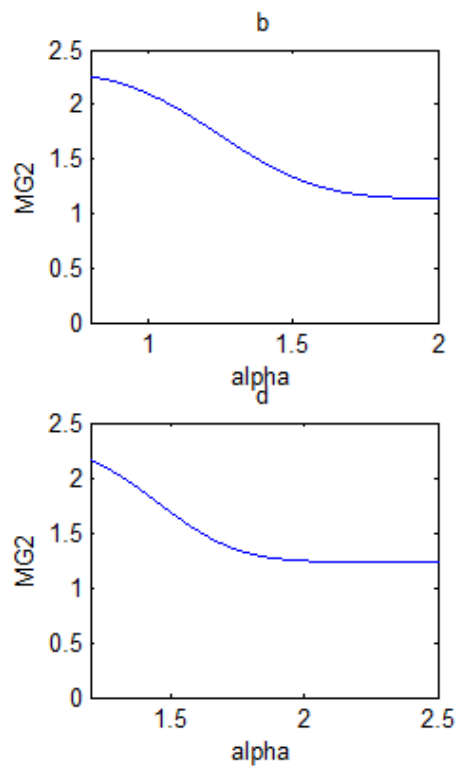

$M_{G}^{2}$ - factor some particular cases.

\section{Conclusion}

On the basis of the generalized truncated second-order moments, generalized $M_{G}^{2}-$ factor of hard-edge diffracted 
circular flattened Gaussian is derived. The closed-form expression for the $M_{G}^{2}-$ factor of hard-edge diffracted CFGB is dependent on the truncation parameter $\alpha$ and beam order $\mathrm{N}$. Two special cases of $\mathrm{M}$-factor of hard-edge diffracted CFGB, one is the case for $\alpha \rightarrow \infty$, for untruncated case, and the other is the case for $\mathrm{N}=1$, for truncated Gaussian beam, have been discussed. In addition, the power fraction is demonstrated analytically and numerically.

\section{References}

[1] N. Nishi, T. Jitsuno, K. Tsubakimoto, S. Matsuoka, N. Miyanaga, M. Nakatsuka, Opt. Rev.7 (2000) 216.

[2] Y. Kato, K. Mima, N. Miyanaga, S. Arinaga, Y. Kitagawa, M. Nakatsuka, C. Yamanaka, Phys. Rev. Lett.53 (1984) 1057.

[3] M.S. Bowers, Opt. Lett.19 (1992) 1319.

[4] Y. Li, Opt. Lett.27 (2002) 1007.

[5] J. Yang. R. Wang Opt. Eng. 42 ( 2003) 3106.

[6] D. Shealy. SPIE.4095 (2000).1
[7] R. Martinez-Herrero, P.M. Mejias, Opt. Lett. 18 (1993) 1669.

[8] R. Martinez-Herrero, P.M. Mejias, M. Arias, Opt. Lett. 20 (1995) 124.

[9] A.E. Siegman, Proc. SPIE 1224 (1990) 2.

[10] P.M. Mejías, H. Weber, R. Martínez-Herrero, A. GonzálezUreña (Eds.), Proceeding of Laser Beam Characterization, Seceded Espanda Optica, Madrid, Spain, 1993.

[11] A.E. Siegman, IEEE J. Quant. Electron. 27 (1991) 1146.

[12] B. Lu *, S. Luo, Opt. Commun. 178 (2000) 275.

[13] M. Ibnchaikh, L. Dalil-Essakali, Z. Hricha, A. Belafhal, Opt. Commun. 190 (2001) 29.

[14] Z. Mei, D. Zhao .APPLIED OPTICS. 44 (2005) 1383.

[15] M. Abramowitch, I. A. Stegun (Eds), Handbook of Mathematical Function, Nath Bureau of Standards, Washington, DC (1964).

[16] .S. Gradshteyn, I.M. Ryznik, Tables of Integrals, Series, and Products, fifth ed., Academic Press, New York, 1994. 